\title{
Modelling and Simulation of Tactile Sensing System of Fingers for Intelligent Robotic Manipulation Control
}

\author{
$\underline{\text { Amir Parsa Anvar }}{ }^{a}$, Amir M. Anvar ${ }^{\text {a }}$, Tien-Fu Lu ${ }^{a}$ \\ ${ }^{a}$ School of Mechanical Engineering, \\ Faculty of Engineering, Computer, and Mathematical Science, \\ The University of Adelaide, \\ Adelaide, South Australia, 5005 \\ AUSTRALIA
}

Email:amir.p.anvar@student.adelaide.edu.au,amir.anvar@adelaide.edu.au,tien-fu.lu@adelaide.edu.au

\begin{abstract}
The robotic manipulation of delicate and sensitive objects is a process that necessitates tactile sensation, sensory feedback and grasping force control. To meet this requirement, work has been done towards the development of a Tactile Sensing System (TSS) for robotic manipulators, using tactile Force Sensing Resistor (FSR) technology. This work includes an experiment towards the determination of the behaviour of the underlying tactile FSR sensor, in response to inputs, the design of an Intelligent System to aid with grasping force control, the combination of hardware components into a Tactile Sensing System, the design of a practical grasping force control algorithm and a grasping force experiment, involving the application of the developed Force Feedback System.
\end{abstract}

In this work, the behaviour of the FSR, in response to an increasing applied force, has been shown. Furthermore, a Fuzzy Expert System Controller that aids with grasping force control has been designed. The systems design of the developed Tactile Sensing System and the comprehensive design of a practical grasping force control algorithm have also been demonstrated.

Distant robotic surgery and assessment on hand following trauma, as a result of disease or surgery, are of the future applications of this work.

Keywords: Force Sensing Resistor, FSR, Tactile Sensing System, TSS, Fuzzy Expert System Controller, Grasping Force Control, Intelligent System, Modelling, Simulation, Manipulator, Tactile 


\section{INTRODUCTION}

This paper presents the work done towards the development of a Tactile Sensing System (TSS) for the application of robotic manipulation, using tactile Force Sensing Resistor (FSR) technology. The problem that this work aims to address is how to support an intelligent-Manipulator robot, with the griping of a delicatesoft-object(s), without damaging it, after the object has been seen and interpreted by the robot brain.

This problem has been approached by setting several objectives. The first objective is on the determination of the behaviour of the underlying tactile FSR sensor in response to inputs. Furthermore, the design of an Intelligent System to aid with grasping force control, the combination of hardware components into a Tactile Sensing System, the basic design for a practical grasping force control algorithm, from evaluation of the normal forces, and the application of the developed Force Feedback System in a grasping force experiment.

Robotic manipulation is a process that is involved with the grasping and movement of objects. Throughout this process, if the acting force on the object is too little or too great, then the object can either slide from the hold of the fingers or face deformation. Therefore, the need for a grasping force control system arises as an issue of importance towards succeeding robotic manipulation.

Previous attempts have been made to develop tactile sensory feedback systems for robotics application (Shimojo et al. 2004), (Park et al. 2012). These systems have been designed to read and display the sensory data, coming from the tactile mechanisms, to the operator. Progress has also been made towards the development of a grasping force control system for robotic manipulators (DeZhang et al. 2005). This system incorporated strain gauge finger force sensors and a multivariable grasping force control algorithm. The work presented by this paper encompasses Force Sensing Resistor technology as an alternative to strain gauge technology. These two forms of technology exhibit different behaviour in terms of force sensing. Altogether, a firm foundation for futuristic development, in the area of Tactile Sensing Systems for grasping force control, has been demonstrated.

The creation of the Tactile Sensing System was for the dual finger prototype robotic manipulator, shown in Figure 1. The region of interest to the design was the fingers, or gripping region, of this prototype.

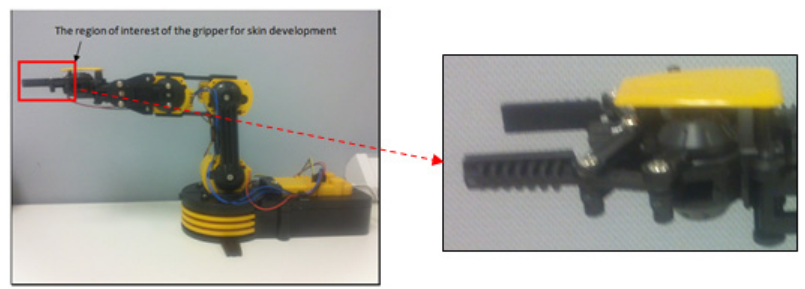

Figure 1. Six degree of freedom prototype manipulator, and its gripping region

The sensor used towards the development of the TSS was the Interlink FSR 400, shown in Figure 2, below.

Figure 2. Images of the front side of an FSR 400 sensor (Interlink Electronics 2010)

\section{SENSOR BEHAVIOUR DETERMINATION EXPERIMENT}

An experiment has been conducted to obtain a reliable relationship between the Force Sensing Resistors input force and output resistance variables. This relationship was required to decode the force value from the resistance reading, in the Force Feedback System.

\subsection{Apparatus and Methodology}

Figure 3 displays and details the apparatus, which has been used in this experiment.

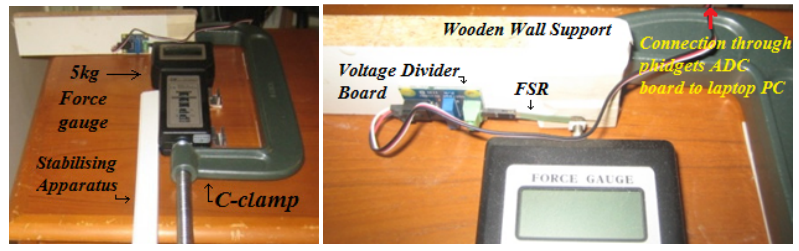

Figure 3. Experimental setup of apparatus 
This experiment was performed as follows. The handle of the C-clamp was wound, to push the force gauge against the surface of the Force Sensing Resistor. Once the desired force reading was displayed on the screen of the force gauge, the corresponding value of the sensor was recorded from a laptop PC. The C-clamp was then unwound and some time was given for the FSR to settle, before the experiment was repeated. This experiment was repeated three times for each desired force value and five times, overall.

\subsection{Calculation of Results}

Each sensor network reading was converted to Voltage by the following equation.

$$
V_{\text {out }}=\text { rawvalue } \times \frac{5}{1000}
$$

Finally, the value of the sensor output Voltage was then converted to resistance, by the voltage divider equation (equation 2).

$$
R_{\text {sensor }}=V_{\text {in }} \frac{R_{\text {fixed }}+R_{\text {variable }}}{V_{\text {out }}}-\left(R_{\text {fixed }}-R_{\text {variable }}\right)
$$

Where: $V_{\text {in }}=5 \mathrm{~V}$ is the input voltage across the voltage divider network,

$\mathrm{R}_{\text {fixed }}=997 \Omega$ is the resistance of the fixed resistor in the voltage divider board,

and $\quad R_{\text {variable }}=19090 \Omega$ is the resistance of the potentiometer in the voltage divider board.

\subsection{Experimental Results and Discussion}

The results of this experiment can be seen in Table 1, below. As can be seen, the value of resistance decreases with an increasing applied force.

Table 1. Experimental results

\begin{tabular}{|l|c|c|c|c|c|}
\hline Force $(\mathrm{g})$ & 12 & 20 & 40 & 70 & 100 \\
\hline Voltage $(\mathrm{mV})$ & 933.3333 & 1719.167 & 2753.333 & 3311.667 & 3715 \\
\hline Resistance $(\mathrm{Ohm})$ & 87521.93 & 38333.75 & 16390.6 & 10240.63 & 6947.993 \\
\hline
\end{tabular}

Furthermore, as can be seen in Figure 4, the relationship between this data is closely modelled by an inverse power law; i.e., $\left(\mathrm{R}^{2}=0.9873\right)$.

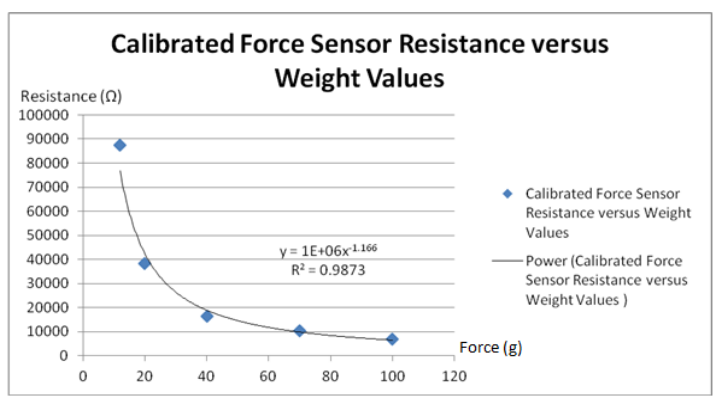

Figure 4. The experimental results of input-output FSR behaviour

The equation for this relationship is presented below.

$$
R_{F S R}=10^{6}\left(F^{-1.166}\right)
$$

Where: $\mathrm{R}_{\mathrm{FSR}}$ is the resistance of the FSR

and $\quad \mathrm{F}$ is the force, which has been applied.

In summary, the behaviour of the FSR, in response to an increasing applied force has been shown to be decreasing, and one that is dictated by an inverse power law. Furthermore, the aim to obtain a reliable relationship between the input force and output resistance variables of the FSR has been acheived. 
Amir Parsa Anvar et al., Modelling and Simulation of Tactile Sensing System of Fingers for Intelligent Robotic Manipulation Control

\section{INTELLIGENT SYSTEM DESIGN}

An Intelligent System has been designed to aid with the grasping force control of the gripper. A scenario has also been presented, which demonstrates its functionality.

\subsection{A Dual Input Fuzzy Expert System Controller}

This Fuzzy Expert System Controller sinks the data from the left and right finger force sensors (SL input and SR input), evaluates it and formulates a decision within its rule base, and presents an output force value $\left(\mathrm{F}_{\text {output }}\right)$ to the grasping force controller (see Figure 5).

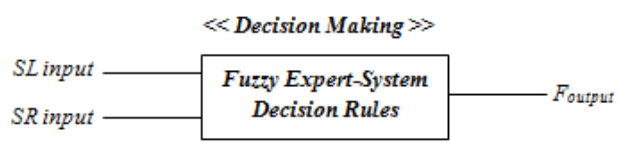

Figure 5. The Fuzzy Expert System Controller Architecture

The rule table of this system can be seen below. It is comprised of three major rules and seven subsidiary rules. The major rules compute the resultant force output on the gripper-wrist, from the sensor-inputs (FSL) and (FSR), by the mathematical theorem of Pythagoras. Based upon this value, the relevant subsidiary rule will then fuzzify the output force to the linguistic value of either 'Low', 'Medium' or 'High'. Therefore, this Fuzzy Expert-System can output the resultant force that the gripper wrist must bear, as well as a classification, to inform the human operator or the automatic controller brain of the status of the object handling, throughout the process of manipulation.

Table 2. Fuzzy Logic Expert-System Rule Base

\begin{tabular}{|c|c|c|}
\hline Rules & $\begin{array}{c}\text { Fuzzy Expert-System } \\
\text { Mixed Chaining - Perception Summary }\end{array}$ & 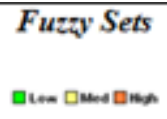 \\
\hline Rule 1 & $\begin{array}{l}\text { IF: } \quad 0.1>F S L>10 \& 0.1>F S R>=10 \\
\text { IHEN } \sqrt{(F S L)^{2}+(\text { FRL })^{2}}=\text { Fout }\end{array}$ & \\
\hline Sub Rule 1.1 & $\begin{array}{ll}\text { IF }: & 0.1>\text { Fout }>=7.5 \\
\text { IHEN }: & \text { Fout }=\text { Min }\end{array}$ & \\
\hline Sub Rule 1.2 & $\begin{array}{ll}\text { IF }: & 7.6>\text { Fout }>=10 \\
\text { IHEN: } & \text { Fout }=\text { Med }\end{array}$ & \\
\hline Rule 2 & $\begin{array}{l}\text { IF: } \quad 5.1>F S L>-15 \& 5.1>F S R>-15 \\
\text { IHEN: } \sqrt{(F S L)^{2}+(F R L)^{2}}=\text { Fout }\end{array}$ & \\
\hline Sub Rule 2.1 & $\begin{array}{ll}\text { IF: } & 5.1>\text { Fout }>=7.5 \\
\text { IHEN: } & \text { Fout }=\text { Min }\end{array}$ & \\
\hline Sub Rule 2.2 & $\begin{array}{ll}\text { IF: } \quad 7.6>\text { Fout }>=12.5 \\
\text { THEN: } & \text { Fout }=\text { Med }\end{array}$ & \\
\hline
\end{tabular}


Amir Parsa Anvar et al., Modelling and Simulation of Tactile Sensing System of Fingers for Intelligent Robotic Manipulation Control

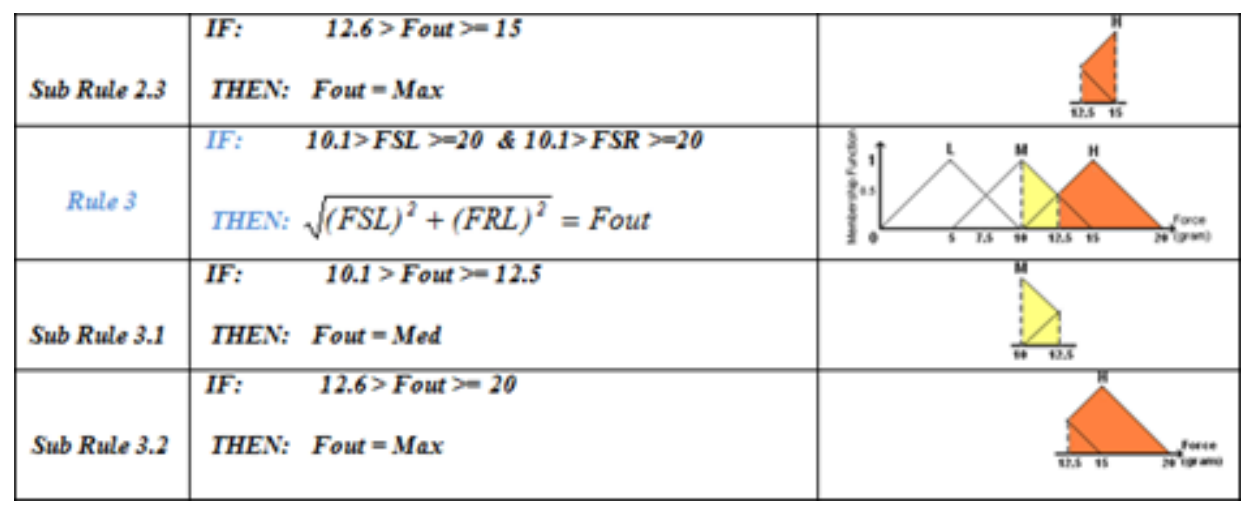

\section{SYSTEMS DESIGN OF THE SENSITIVE ARTIFICIAL SKIN SYSTEM}

The system design for the developed Tactile Sensing System is shown in Figure 6. As can be seen, this design comprises two Force Sensing Resistors, which have been bound to the fingers of the manipulator, by double sided adhesive tape. Each FSR is connected to a voltage divider, and each divider has a potentiometer for sensitivity adjustment. The range of each force sensitive resistor is from 0.01 kilograms to 10 kilograms. The potentiometers have been wound up to their greatest extent, making the system highly sensitive to forces within the range of 0.1 grams. Each voltage divider is connected to a port of the phidgets Analogue to Digital Converter board, which converts the analogue voltage across the (potentiometer and fixed resistor) in the voltage divider board to a digital value that is then sent to a Computer-Controller, via USB. The ComputerController only contains a program that gathers the raw readings of the left and right finger FSRs, decodes them into force values and prints them out, along with a time stamp. Hence, the developed System is currently a Force Feedback System. However, the Computer-Controller is intended to contain the grasping force control algorithm, that informs the gripper to grasp the object and, based upon the converted force readings from the FSRs, makes a decision to either halt or continue grasping. The details of this algorithm have been presented within the next section (5).

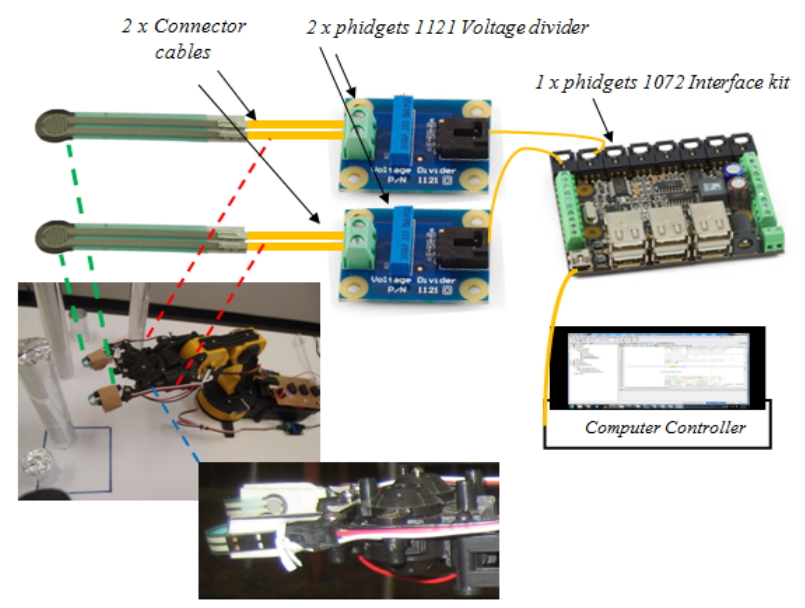

Figure 6. Architecture of the developed Tactile Sensing System

\section{THE BASIS FOR THE DESIGN OF A PRACTICAL GRASPING FORCE CONTROL ALGORITHM}

The Intelligence aspect of the TSS has been addressed by the basic design of an algorithm that conducts grasping force control for the prototype manipulator. This design, labelled "IFKv2.java", can be seen in Figure 7. It functions based upon the principle that, if either the left force sensor or the right force sensor deliver a value that is above the known threshold that is required to hold the object, the controller must then halt grasping. 


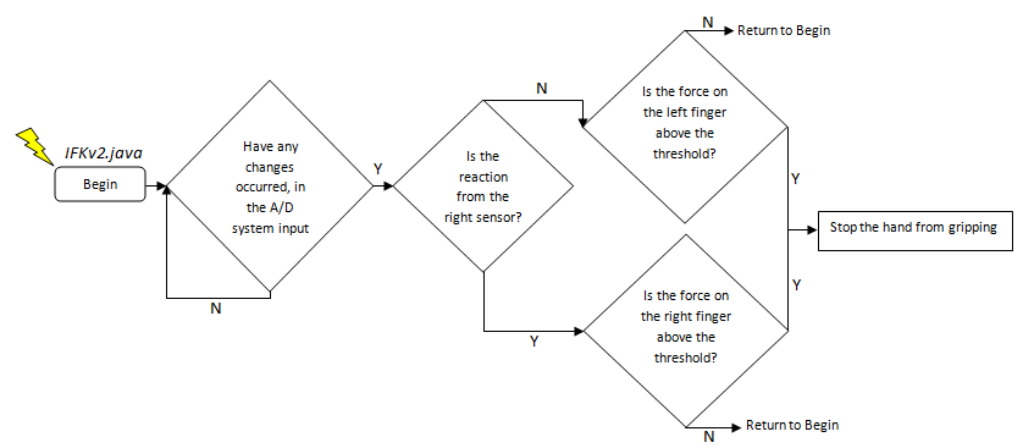

Figure 7. Finite state flow diagram of the grasping force control algorithm

\section{GRASPING FORCE EXPERIMENT}

An experiment has been conducted to test the developed Force Feedback System. The objective of this experiment was to obtain the force feedback of the sensors, from grasping the soft surface of a delicate aluminium object. The purpose of this experiment was to use the obtained data for further automation, and to train intelligent grasping force control algorithms.

\subsection{Experimental Apparatus and Procedure}

The manipulator was controlled by means of two programs. These were the Arduino Program and the Processing Program. The Arduino Program acted as an interface between the robot and the robotic control program on the computer. This control program was the Processing Code that served as a Human Computer Interface in the manipulation of the robot; i.e., it provided buttons to move the joints of the manipulator.

The experiment was conducted by grasping an object, moving it to a desired position and releasing the object; whilst rapidly recording force measurements (See Figure 8).

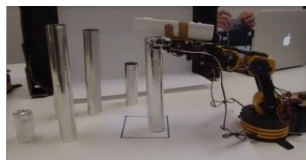

(a)

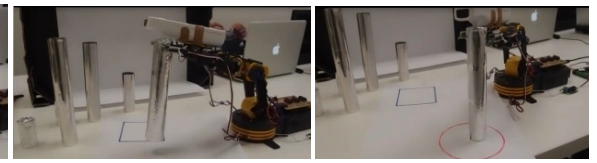

(b)

(c)

Figure 8. Time series transition images of the grasping experiment. (a) Grasping the object, (b) Moving the object (c) Releasing the object

\subsection{Experimental Results and Discussion}

The results of this experiment have been presented in the form of a graph, shown in Figure 9. As can be seen, at zero seconds, the average value of the left and right finger force sensors is 1.6 grams. From zero to thirteen seconds, the manipulator grasps the object, which can be seen as a progressive rise in the curve. From thirteen to one hundred seconds, the object is moved from the location of the blue square to the location of the red circle. Throughout this phase, the average value of the sensors remains near constant. This can be seen as an almost steady line along eighty percent of the way. The variations in the average value are possibly due to vibrations within the manipulator. From one hundred seconds to one hundred and fourty one seconds, the object is released from the hold of the gripper fingers, and therefore, the progressive drop in the average value of the finger force sensors to zero.

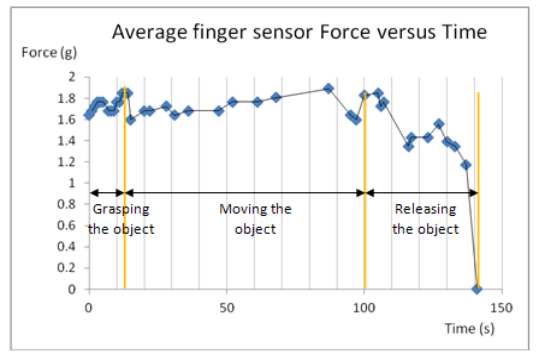

Figure 9. Force feedback from the grasping and manipulation experiment 
The lesson learnt from this experiment is that its results were near accurate and therefore useful for training intelligent grasping force control algorithms.

\section{CONCLUSIONS}

The aim of this paper was to present the work done towards the development of a Tactile Sensing System for the application of robotic manipulation, using tactile Force Sensing Resistor technology, to support an independent intelligent-Manipulator robot with the safe griping of a delicate-soft-object(s), after the object has been seen and interpreted by the robot brain. This aim has been approached from several objectives.

Firstly, the process behind the determination of the behaviour of the underlying tactile Force Sensing Resistor, in response to inputs, and the results of this experiment have been demonstrated. The behaviour of the FSR, in response to an increasing applied force, has been shown to be decreasing, and one that is dictated by an inverse power law. A reliable relationship has been obtained between the input force and output resistance variables of the FSR. This relationship is fundemental towards the development of the TSS, as it plays the role of decoding the force value, from the corresponding resistance reading, of an FSR finger sensor.

Secondly, the design of an Intelligent System, that aids with grasping force control, has been demonstrated. This Fuzzy Expert System Controller has been designed to present both a numerical and linguistic output of the resultant force, acting on the wrist of the gripper, from knowledge of the left and right finger forces. This output is useful for presentation to the human operator or the grasping force control loop of the TSS, to address the problem of how to support the manipulator robot, with the safe griping of an object.

This paper has also demonstrated the systems design of a developed Tactile Sensing System, which comprises Force Sensing Resistors as the tactile finger force sensing mechanisms, voltage dividers for force measurement sensitivity adjustment and an Analogue to Digital Convertor board for data conversion and fusion into a Computer-Controller. Although the development of a working grasping force control program is still in progress, the basic logical design of this algorithm has been demonstrated.

Finally, the procedure and results of a grasping force experiment, demonstrating the application of the developed Force Feedback System, have been presented. The results of this experiment were near accurate and therefore useful for training intelligent grasping force control algorithms of Tactile Sensing Systems.

A recommendation for further research is to study the use of neural networks towards the implementation of a grasping force control algorithm. Neural networks show great potential for the application of grasping force control, as they are capable of being trained with the data that is required to successfully grasp a specified object and can 'learn' from the mistakes made in grasping.

Future applications of this work are distant robotic surgery, as well as assessment on hand following trauma, as a result of disease or surgery.

\section{REFERENCES}

DeZhang, X, Ge, YJ, Fei, S, Cheng, WZ, LiFu, G \& Yuman, N 2005, 'Tactile Sensing for Underwater Operation System Based on Multi Finger Sensors Information Fusion', in Proceedings of the 2005 IEEE International Conference on Information Acquisition, Hong Kong and Macau, pp. 26-31.

Interlink Electronics 2010, FSR 400 Data Sheet, Interlink Electronics, Sensor Technologies, viewed September 3 2012, <http://www.trossenrobotics.com/productdocs/2010-10-26-DataSheet-FSR400Layout2.pdf $>$.

Park, Y-L, Chen, B-R, Wood R \& J 2012, 'Design and Fabrication of Soft Artificial Skin Using Embedded Microchannels and Liquid Conductors', IEEE Sensors Journal, vol. 12, no. 8, pp. 2711-2718.

Shimojo, M, Namiki A, Ishikawa M \& Makino, R 2004, 'A Tactile Sensor Sheet Using Pressure Conductive Rubber With Electrical-Wires Stitched Method’, IEEE Sensors Journal, vol. 4, no. 5, pp. 589-596. 